\title{
Atividade física como prevenção da obesidade e síndrome metabólica na infância e adolescência: uma revisão integrativa
}

\author{
Marcília Ingrid Lima Barroso Nunes ${ }^{\mathrm{a}}$ (D) , André Gustavo de Medeiros Matos ${ }^{\mathrm{bc}}$ (D), Leonardo Emmanuel de \\ Medeiros Limad ${ }^{\text {(iD) }}$, José Garcia De Brito-Neto ${ }^{\text {(iD }}$ \\ aUniversidade Potiguar (UnP), Mossoró, RN, Brasil. \\ ${ }^{\mathrm{b}}$ Faculdade Nova Esperança (FACENE/RN), Mossoró, RN, Brasil. \\ cUniversidade Paulista (UNIP), Mossoró, RN, Brasil. \\ dCentro Universitário de Jaguariúna (EAD UNIFAJ - UNIEDUK), Jaguariúna, SP, Brasil. \\ eLiga Acadêmica Multidisciplinar de Oncologia da FACENE (LAMOF), Faculdade Nova Esperança (FACENE/RN), Mossoró, RN, Brasil.
}

RESUMO A prevalência da obesidade em crianças e adolescentes tem apresentado um rápido aumento durante as últimas décadas, sendo caracterizada como uma verdadeira epidemia mundial e desempenhando papel central no desenvolvimento da síndrome metabólica (SM), também é conhecida como síndrome $X$ sendo definida como um transtorno complexo, cinco fatores de risco preenchem os critérios para o seu diagnóstico: aumento da circunferência da cintura (CC), baixo nível de lipoproteína de alta densidade (HDL), triglicérides elevados (TG), elevados pressão arterial (PA) e glicemia de jejum. Trata-se de um estudo de revisão integrativa da literatura, que contribui para o processo de sistematização e análise dos resultados de outros estudos independentes sobre o tema abordado. Esta revisão visa ainda analisar a prevalência de sobrepeso e obesidade na infância e adolescência e sua relação com a síndrome metabólica e fatores que podem intervir positivamente no quadro. Dentre os achados foram selecionados 31 artigos entre os anos de 2000 e 2019 que associam a SM a um conjunto de alterações clínicas e laboratoriais, apontando ainda que a prática regular do exercício físico pode auxiliar na prevenção e/ou até minimizar os efeitos deletérios da síndrome metabólica, gerando resultados promissores quando a dieta é associada a prática regular de atividade física. Os benefícios da prática regular da atividade física em jovens vão além da perda de peso, atuando como uma terapia complementar não farmacológica, refletindo nos parâmetros metabólicos, bem-estar psíquico além da predisposição para manter a atividade física na idade adulta e, por consequência, diminuição de risco para doença cardiovascular, aumentando ainda a expectativa de vida do indivíduo.

\section{PALAVRAS-CHAVE exercício físico, obesidade, síndrome metabólica, sobrepeso}

Aceito 23 de fevereiro de 2021 Publicado online 23 de março de 2021

Cite este artigo:

Nunes et al. (2021) Atividade física como prevenção da obesidade e síndrome metabólica na infância e adolescência: uma revisão integrativa. Multidisciplinary Reviews 4 : e2021009. DOI: 10.29327/multi.2021009.

\section{The use of metformin in the treatment of Gestational diabetes mellitus}

ABSTRACT The prevalence of obesity in children and adolescents has shown a rapid increase during the last decades, being characterized as a true worldwide epidemic and playing a central role in the development of metabolic syndrome (MS), it is also known as syndrome X. This complex disorder has five risk factors meet the criteria for its diagnosis: increased waist circumference (WC), low level of high-density lipoprotein (HDL), high triglycerides (TG), high blood pressure (BP) and fasting blood glucose. Thus, this integrative review study contributes to systematization and analysis of the results from other independent studies on the subject addressed. This review yet aims to analyze the prevalence of overweight and obesity in childhood and adolescents and its relationship with metabolic syndrome and factors that can positively intervene in the condition. Among the findings, 31 articles were selected between the years or 2000 and 2019 that associate the MS with a set of clinical laboratory changes, pointing out that regular physical exercise can help 
prevent and/or even minimize the harmful effects of the metabolic syndrome. The results are promising when a diet is associated with a regular practice of physical activity. The benefits of a regular exercise of physical activity in young people go beyond the weight loss, acting as a complementary non-pharmacological therapy, reflecting on metabolic parameters, psychic well-being in addition to the predisposition to maintain physical activity in adulthood. Therefore, it decreased the risk for cardiovascular disease, further increasing the individual's life expectancy.

KEYWORDS: obesity, overweight, metabolic syndrome, physical exercise

\section{Introdução}

A prevalência da obesidade em crianças e adolescentes tem apresentado um rápido aumento durante as últimas décadas, sendo caracterizada como uma verdadeira epidemia mundial, estando ainda associada a diversos problemas de saúde e, em particular, desempenha papel central na síndrome metabólica - SM (Abrantes et al 2002; Oliveira 2003; Brandão et al 2005; Braga 2006; Buff et al 2007; Teixeira et al 2009; Tavares et al 2010; Lucena et al 2017; Silva et al 2019).

A obesidade é o aumento do tecido adiposo, e é responsável por um estado pró-inflamatório, causando o aumento de liberação de citocinas (interleucinas e fator de necrose tumoral alfa) e diminuição de produção de adiponectina, refletindo numa diminuição da sensibilidade à insulina (Balaban e Silva 2001; Damiani et al 2011; França et al 2017).

A etiologia da obesidade é claramente identificada como sendo multifatorial, sociólogos e nutrólogos explicam esse aumento estatístico nos últimos anos devido aos fatores nutricionais inadequados (excessivo consumo de lanches, guloseimas, etc) associados a um excessivo sedentarismo (TV, vídeo-games, etc) (Diamini 2000; Oliveira 2003; Silva et al 2005; Buff et al 2007; Rodrigues et al 2009; Silva et al 2019).

Em 1988, Reaven descreveu síndrome metabólica (SM) como um conjunto de alterações clínicas e laboratoriais associadas a risco aumentado de doença cardiovascular. Diversos estudos apontam não haver consenso no que diz respeito aos componentes da síndrome, sabe-se que a hiperinsulinemia e a resistência à insulina (RI) estão frequentemente presentes e são fatores centrais para o desenvolvimento de complicações (Tavares et al 2010; Braga 2006; Fernandes et al 2009; Teixeira et al 2009; Rodrigues et al 2009; França et al 2017).

A síndrome metabólica, também é conhecida como síndrome $X$ ou ainda como síndrome da resistência à insulina. O termo síndrome metabólica foi sugerido pela Organização Mundial de Saúde (OMS), visto que não foi comprovado que a resistência à insulina poderia ser o fator causal comum (Souza et al 2007; Mella 2012; Tibana e Prestes 2013; Nascimento et al 2016).

Fatores de risco que são identificados como SM: sobrepeso e/ou obesidade, principalmente se houver acúmulo de gordura predominante na região abdominal; dislipidemia; hipertensão arterial; aumento de triglicérides, diminuição do HDL colesterol, história pessoal de intolerância à glicose ou diabetes gestacional; síndrome dos ovários policísticos (SOP) são frequentemente encontrados nesses indivíduos. No entanto, estudos recentes sugerem que a SM pode ter início ainda na vida intrauterina, podendo também ser observado no acompanhamento de crianças nascidas pequenas para a idade gestacional (PIG) (Brandão et al 2005; Braga 2006; Duarte et al 2015).

O diagnóstico da SM ainda não se encontra bem definido em crianças e adolescentes, devido a falta de um consenso internacional, porém existe o alerta para as alterações iniciais de cada um desses fatores surgindo em associações variadas, e embora sejam de pequena expressão determinam um perfil cardiovascular não favorável para esses jovens (Brandão et al 2005; Souza et al 2007).

Damiani (et al 2011) complementa afirmando que o diagnóstico da SM na infância e na adolescência é problemático, uma vez que pressão arterial (PA), perfil lipídico e valores antropométricos variam de acordo com a idade e o estado puberal, devendo ser usado, portanto, diferentes pontos de corte para sexo e idade para cada uma das variáveis.

Pesquisas apontam grande correlação entre obesidade e prevalência de SM independente de quais critérios sejam utilizados. Esse fato, junto à epidemia atual de obesidade em crianças e adolescentes, leva-nos a crer num 
desproporcional aumento de doenças cardiovasculares ainda na infância (Ciolac e Guimarães 2004; Rodrigues et al 2009; Diamini et al 2011). Fato preocupante uma vez que doenças cardiovasculares apresentadas durante a infância têm grandes possibilidades de se manterem até a vida adulta e predizem o desenvolvimento de síndrome metabólica assim como uma série de comorbidades no futuro (Balaban e Silva 2001; Abrantes et al 2002; Oliveira et al 2004; Braga 2006; Buff et al 2007; Teixeira et al 2009; Tavares et al 2010; Loureiro Neto et al 2019).

Diversos estudos afirmam haver forte associação entre obesidade e sedentarismo, assim como relatam associação inversa entre atividade física, uma vez que o comportamento de movimento dos indivíduos pode impactar no controle dos componentes da síndrome metabólica, esses estudos discorrem sobre os benefícios da atividade física sobre a obesidade em seus diversos tipos de intensidade, indicando que a manutenção de um estilo de vida ativo, independente de qual atividade praticada, pode auxiliar na prevenção dessa doença assim como reduzir os fatores de risco metabólico na infância e adolescência (Ciolac e Guimarães 2004; Buff et al 2007; Loureiro Neto et al 2019).

Sendo assim o presente estudo tem como objetivo analisar a prevalência de sobrepeso e obesidade na infância e adolescência e sua relação com a síndrome metabólica, assim como fatores que podem intervir positivamente no quadro.

\section{Métodos}

Trata-se de um estudo de revisão integrativa da literatura, uma vez que ela contribui para o processo de sistematização e análise dos resultados, visando a compreensão de determinado tema, a partir de outros estudos independentes.

Foram adotados os seguintes critérios de inclusão: estudos com textos completos disponíveis para análise e que estejam indexados eletronicamente nos sites PubMed e SciELO; artigos na língua portuguesa e/ou inglesa; artigos que abordassem uma das variáveis estudadas, tais como composição corporal, perfil glicêmico, perfil lipídico, níveis de insulina, hipertensão arterial, sedentarismo, nível de atividade física; pesquisas com amostra composta por crianças e/ou adolescentes com sobrepeso ou obesidade, entre os anos de 2000 e 2019. Os termos de pesquisa utilizados foram: obesidade, sobrepeso, síndrome metabólica, exercício físico.

Os critérios de exclusão adotados neste estudo foram: artigos não disponíveis gratuitamente; artigos disponíveis apenas em resumo; publicações como cartas, comentários e editoriais.

Do material obtido, 47 artigos, procedeu-se à leitura minuciosa de cada resumo/artigo, destacando aqueles que responderam ao objetivo proposto por este estudo, a fim de organizar e tabular os dados. Seguindo os critérios de inclusão, 30 estudos foram selecionados para análise, os quais são referenciados no presente texto.

As buscas foram realizadas no período de 25 de abril de 2020 a 05 de junho de 2020, a triagem ocorreu de 25 de abril de 2020 a 05 de junho de 2020, com a leitura integral dos artigos obtidos.

\section{Resultados e Discussão}

Os estudos selecionados foram classificados quanto à sua categoria de publicação, conforme explicitado pelos periódicos, assim especificados: 51,61\% (16) pesquisas originais, 48,38\% (15) estudos de revisão de literatura.

Em relação aos anos de publicação dos artigos, compreendidos entre 2000 e 2019, os dados coletados apresentaram a distribuição, a seguir, apresentada na Figura 1.

$\mathrm{Na}$ classificação dos estudos, quanto ao contexto e suas correlações, 31 (100\%) estudos descrevem síndrome metabólica (SM) como sendo um conjunto de alterações clínicas e laboratoriais, 31 (100\%) associam ao risco aumentado de doença cardiovascular, 27 (87,09\%) apresentam forte relação com o perfil glicêmico do indivíduo, 25 (80,64\%) alertam riscos do desenvolvimento precoce de diabetes mellitus tipo 2 (DM2), 14 (45,16\%) relacionam o sobrepeso ao nível socioeconômico, 28 (90,32\%) dos estudos apontam a inatividade física/sedentarismo como sendo um dos fatores de risco para o desenvolvimento da SM, 26 (83,87\%) afirmam que a prática regular do exercício físico pode auxiliar na prevenção e/ou até minimizar os efeitos deletérios da síndrome metabólica, e 23 (74,19\%) apontam resultados promissores quando a dieta é associada a prática regular de atividade física. 


\section{Obesidade na infância e adolescência}

Diversos fatores têm importância na gênese da obesidade. Podemos citar como exemplo os fatores genéticos, os fisiológicos e os metabólicos; no entanto, no tocante a este crescente aumento do número de indivíduos obesos parecem estar mais relacionados as mudanças no estilo de vida e aos hábitos alimentares (Oliveira e Fisberg 2003; Mella 2012; Tibana e Prestes 2013; Lourenço Neto et al 2019).

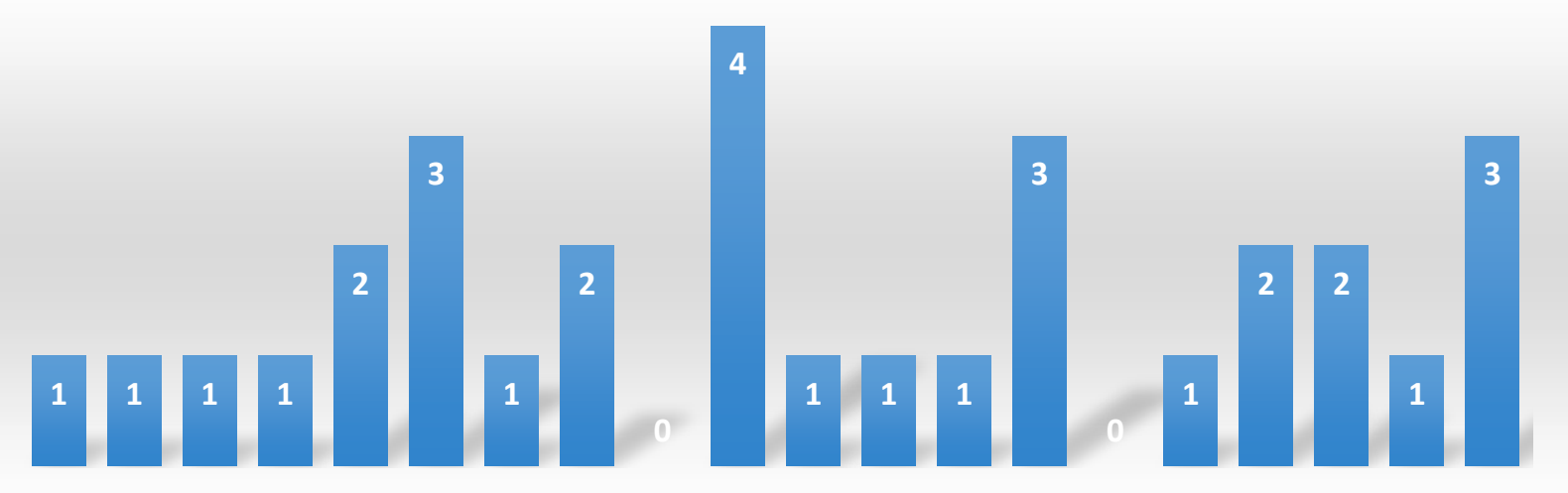

20002001200220032004200520062007200820092010201120122013201420152016201720182019

Figura 1 Quantitativo de estudos estratificados segundo ano de publicação.

Estudos recentes afirmam que no mundo, cerca de 42 milhões de crianças menores de cinco anos de idade apresentam sobrepeso. Mudanças comportamentais e ambientais como a adoção de uma dieta ocidentalizada e estilo de vida sedentário, seguindo o aumento socioeconômico dos países (Balaban e Silva 2001; Silva et al 2005; Mella 2012; Lourenço Neto et al 2019).

Nos últimos anos, os estudos científicos "redescobriram" o tecido adiposo, atestando que o mesmo passa da condição de um mero depositário da gordura corporal que tenha a finalidade de proteger o indivíduo contra as baixas temperaturas ambientais (Damiani 2000; Siqueira et al 2016).

A presença de sobrepeso/obesidade aparece como o mais importante e presente fator de risco dentre todos os que fazem parte da síndrome metabólica (SM), reforçando o que pesquisadores apontam de que a obesidade, por si só, seria um determinante expressivo da síndrome metabólica, ainda que durante a infância e a adolescência (Brandão et al 2005; Braga 2006; Buff et al 2007; França et al 2017; Lourenço Neto et al 2019; Paz et al 2019).

Atualmente os jovens estão praticando menos atividade física, tendendo a ficar dentro de casa "presos" à televisão, videogames e computadores. A insegurança das grandes cidades contribui com tal evento, de forma que inibem atividades ao ar livre, como caminhadas, andar de bicicleta em ruas e parques, resultando em um conjunto de ações que estão nos direcionando para modificações no grupo familiar como um todo (Brandão et al 2005; Silva et al 2005; Tibana e Prestes 2013; França et al 2017).

Realidade preocupante, uma vez que a criança obesa nos primeiros seis meses de vida tem 2, 3 vezes mais possibilidade de se tornar um adulto obeso, enquanto adolescentes obesos entre os 10 aos 13 anos de idade têm 6,5 vezes mais possibilidade de se tornarem adultos obesos. Em crianças com menos de 10 anos de idade, as alterações cardiometabólicas podem ser sutis, surgindo lenta e progressivamente (Damiani 2000; Balaban e Silva 2001; Damiani et al 2011).

Desta forma, podemos observar claramente a importância de detectar precocemente tais alterações em crianças e adolescentes que estejam acima do peso, independente de histórico familiar de doença cardiovascular precoce e/ou diabetes tipo 2 e tentar modificar seus hábitos diários para que estes possam ter uma melhora qualitativa e quantitativa em sua vida (Damiani 2000; Oliveira e Fisberg 2003; Damiani et al 2011; Duarte et al 2015). 


\section{Síndrome metabólica na juventude}

A síndrome metabólica (SM), também conhecida como a síndrome X, é definida como um transtorno complexo, segue afetando populações cada vez mais jovens e se caracteriza por um conjunto de fatores de risco cardiovasculares. São cinco os fatores de risco que preenchem os critérios para o diagnóstico da SM: aumento da circunferência da cintura (CC), baixo nível de lipoproteína de alta densidade (HDL), triglicérides elevados (TG), elevados pressão arterial (PA) e glicemia de jejum (Ciolac e Guimarães 2004; Souza et al 2007; Mella 2012; Tibana e Prestes 2013; Marques et al 2018; Lourenço Neto et al2019).

Além de riscos cardiovasculares, péssimos hábitos de vida podem estar associados ao desenvolvimento, como o consumo de álcool, o tabagismo, ingestão de alimentos com alto valor calórico, inatividade física e o sedentarismo, resultando numa quantidade excessiva de triglicérides presente no corpo e no seu armazenamento em locais específicos, como o fígado, adipócitos e nos músculos, gerando uma alta atividade do tecido adiposo, que são associados a um maior risco de desenvolvimento da SM (Ciolac e Guimarães 2004; Damiani et al 2011; Nascimento et al 2016; Marques et al 2018; Lourenço Neto et al 2019).

Devido a dificuldade de seu diagnóstico, a Organização Mundial da Saúde (OMS) propôs o diagnóstico da SM em indivíduos que venham a apresentar evidências de resistência à insulina e a, pelo menos, mais dois fatores de risco cardiovascular. O principal objetivo de critérios arbitrários serem estabelecidos para o diagnóstico da SM é a possibilidade de identificar indivíduos com alto risco de complicações e utilizar essa informação para dar início a modificações no estilo de vida objetivando a melhora do prognóstico dessas pessoas (Abrantes et al 2002; Braga 2006; Rodrigues et al 2009; Teixeira et al 2009; Del Vecchio et al 2013; Lourenço Neto 2019).

Existe uma falta de um consenso internacional quanto ao diagnóstico preciso da SM em crianças e adolescentes, porém alertam para as alterações iniciais de seus fatores surgindo em associações variadas de três ou mais fatores de risco para diagnóstico da SM (Brandão et al 2005; Braga 2006; Souza et al 2007; Tavares et al 2010; Lourenço Neto et al 2019).

A IDF - International Diabetes Federation - foi a primeira organização a apresentar uma definição de SM específica para jovens, apresentando tal definição de SM para o uso em crianças e adolescentes, utilizando, como um dos critérios, a obesidade abdominal, ressaltando ainda suas peculiaridades e a importância de estudos direcionados a essa faixa etária (IDF, 2007).

Estudos sugerem que o estudo dos distúrbios metabólicos nas crianças devem utilizar uma cautelosa abordagem em etapas, levando em consideração o histórico da criança (faixa e adequação do peso ao nascer, tempo de amamentação), histórico familiar (antecedentes de doença cardiovascular - DCV e DMII) e etnia, associando aos dados clínicos e metabólicos atuais, tal abordagem permitiria definir melhor o risco e indicar possíveis intervenções de forma eficaz (Brambilla et al 2007; Rodrigues et al 2009; França et al 2017).

Elucidar as relações da SM e seus componentes com a prática regular da atividade física em crianças e adolescentes pode mostrar caminhos para o delineamento de estratégias promissoras promotoras da saúde cardiometabólica desse público vulnerável (Oliveira et al 2004; França et al 2017; Lourenço Neto et al 2019).

\section{Exercício físico como terapia complementar}

Nas últimas décadas temos observado um rápido e crescente aumento no número de pessoas obesas, tornando então a obesidade um problema de saúde pública. Estudos sugerem que grande parte da obesidade tem maior ligação ao baixo gasto energético que ao alto consumo de comida, visto que a inatividade física da vida moderna parece ser o maior fator etiológico do aumento dessa doença nas sociedades industrializadas (Ciolac e Guimarães 2004; Oliveira et al 2004, Braga 2006; Damiani et al 2011).

A inatividade física e o sedentarismo são apontados como fatores de risco envolvidos na prevalência da síndrome metabólica (SM), elevando as chances do surgimento da resistência à insulina, obesidade central, aumento do IMC, triglicerídeos e elevada taxa de glicose (Oliveira et al 2004; Brandão et al 2005; Silva et al 2005; Oliveira et al 2009; Fernandes et al 2009; Lourenço Neto et al 2019). 
Duas vias podem ser usadas no tratamento para a SM, podendo ser realizado através de intervenção medicamentosa ou ainda não medicamentosa. Ao se optar pelo tratamento medicamentoso, deve-se levar em consideração aspectos como: horário para administração dos medicamentos, os tipos de fármacos utilizados, o tempo de utilização do medicamento, o objetivo terapêutico que o medicamento prevê entre outros. Ao optar pelo tratamento não medicamentoso, inicialmente o paciente deve ser orientado sobre a importância de se adotar um estilo de vida saudável, com a inclusão da prática de exercícios físicos regular associando a uma dieta balanceada. Neste tipo de tratamento observou-se que o exercício físico apresenta um papel de extrema importância (Del Vecchio et al 2013; Tibana e Prestes 2013; França et al 2017; Marques et al 2018; Paz et al 2019).

Estudos demonstraram que os benefícios da atividade física sobre a obesidade podem ser alcançados, independente de qual atividade esteja sendo praticada e/ou nível de intensidade esteja sendo aplicado, funcionando como uma terapia não medicamentosa, trabalhando sempre pontos determinantes como a aptidão cardiorrespiratória, elevando o bem estar corporal do indivíduo além de fornecer qualidade de vida tanto física como mental (Ciolac e Guimarães 2004; Brandão et al 2005; Fernandes et al 2009; Duarte et al 2015; Lucena et al 2017; Silva et al 2019; Paz et al 2019).

Relações entre os comportamentos de movimentos indicadores de saúde em crianças e jovens afirmam que o tempo gasto em atividade física sendo ela de moderada a vigorosa está fortemente associado à diminuição de marcadores cardiometabólicos (Ciolac e Guimarães 2004; Silva et al 2005; Souza et al 2007; Fernandes et al 2009; Del Vecchio et al 2013; Nascimento et al 2016; Lourenço Neto et al 2019).

Pesquisas quanto ao efeitos do treinamento resistido sobre o metabolismo ainda tem menor número quando comparamos com as que envolvem exercícios aeróbios, por serem atividade que envolvem grandes grupos musculares, no entanto, com base em revisões recentes, autores propõem que um programa de atividade física que esteja focalizado na prevenção e tratamento da SM, deve incluir componentes que melhorem o condicionamento cardiorrespiratório, força e resistência muscular (Ciolac e Guimarães 2004; Mella 2012; Pereira Junior et al 2013; Duarte et al 2015; Silva et al 2019).

Medidas não medicamentosas devem ser a primeira atitude a ser tomada, destacando a promoção do aumento da atividade física assim como a implantação de programas de exercício físico como forma de terapia não farmacológica, objetivando uma mudança centrada em um estilo de vida ativo associado a uma dieta balanceada, tendo visto que o gasto calórico proveniente da sua prática regular mostrou-se imprescindível na perda de massa corporal, sendo também fator auxiliar para o aumento nos níveis de aptidão física, impactando no controle dos componentes da SM, como redução significativa da gordura abdominal, peso corporal, IMC, CA, níveis de glicose sanguínea e TG, normalização dos níveis pressóricos e níveis adequados de colesterol HDL (Ciolac e Guimarães 2004; Oliveira et al 2004; Brandão et al 2005; Souza et al 2007; Oliveira et al 2009; Fernandes et al 2009; Del Vecchio et al 2013; Pereira Junior et al 2013; Duarte et al 2015; França et al 2017; Lucena et al 2017; Lourenço Neto et al 2019).

\section{Considerações finais}

Podemos concluir que a obesidade na infância e adolescência é um fator de risco impactante para o desenvolvimento das doenças cardiovasculares na vida adulta. Sugere-se a atividade física e dieta como prioridade máxima na prevenção específica da obesidade, visto que o seu êxito poderá repercutir de forma direta e positiva na melhora da dislipidemia, hipertensão arterial e nas alterações do metabolismo dos carboidratos.

Os benefícios da prática regular da atividade física em jovens vão além da perda de peso, atuando como uma terapia complementar não farmacológica, refletindo nos parâmetros metabólicos, redução da pressão arterial e da resistência à insulina, bem-estar psíquico, predisposição para manter a atividade física na idade adulta e, por consequência, diminuição de risco para doença cardiovascular, aumentando ainda a expectativa de vida do indivíduo.

\section{Declaração de conflito de interesse}

Os autores declaram que não há conflitos de interesse. 


\section{Referências}

Abrantes MM, Lamounier JA, Colosimo EA (2002) Prevalência de sobrepeso e obesidade em crianças e adolescentes das regiões Sudeste e Nordeste. Jornal de Pediatria 78:335-340.

Balaban G, Silva GAP (2001) Prevalência de sobrepeso em crianças e adolescentes de uma escola da rede privada de Recife. Jornal de Pediatria 77:96-100.

Braga C (2006) Síndrome metabólica na adolescência. Adolescência \& saúde 3:18-20.

Brambilla P, Lissau I, Flodmark CE, Moreno LA, Widhalm K, Wabitsch M, Pietrobelli A (2007) Metabolic risk-factor clustering estimation in children: to draw a line across pediatric metabolic syndrome. International Journal of Obesity 31:591-600.

Brandão AP, Brandão AA, Berenson GS, Fuster V (2005) Metabolic syndrome in children and adolescents. Arquivos Brasileiros de Cardiologia 85:79-81.

Buff CG, Ramos E, Souza FI, Sarni RO (2007) Frequência de síndrome metabólica em crianças e adolescentes com sobrepeso e obesidade. Revista Paulista de Pediatria 25:221-226.

Ciolac EG, Guimarães GV (2004) Exercício físico e síndrome metabólica. Revista Brasileira de Medicina do Esporte 10:319-324.

Damiani D (2000) Obesidade na Infância e Adolescência - Um Extraordinário Desafio! Arquivos Brasileiros de Endocrinologia e Metabolismo 44:363-365.

Damiani D, Kuba VM, Cominato L, Damiani D, Dichtchekenian V, Menezes Filho HC (2011) Síndrome metabólica em crianças e adolescentes: dúvidas na terminologia, mas não nos riscos cardiometabólicos. Arquivos Brasileiros de Endocrinologia e Metabolismo 55:576-582.

Del Vecchio FB, Galliano LM, Coswig VS (2013) Aplicações do exercício intermitente de alta intensidade na síndrome metabólica. Revista Brasileira de Atividade Física 18:669-687.

Duarte AS, Christofoli MC, Pontin B, Paludo J (2015) Síndrome metabólica na infância e adolescência: uma revisão. Revista Brasileira de Nutrição Clínica 30:170-173.

Fernandes RA, Sponton CHG, Zanesco A (2009) Atividade Física na Infância e na Adolescência Promove Efeitos Benéficos na Saúde de Adultos. Revista Sociedade Brasileira de Cardiologia 22:365-372.

França ML, Souza SS, Marques NSF (2017) Benefícios da prática de exercício físico em pacientes com síndrome metabólica. Revista DêCiência em Foco 1:30-47.

IDF - International Diabetes Federation. The IDF consensus definition of the metabolic syndrome in Children and adolescents. Brussels-Belgium, 2007.

Loureiro Neto FM, Borges JWP, Moreira TMM, Machado MMT, Gonzalez RH (2019) Síndrome metabólica e a atividade física em adolescentes: uma revisão integrativa. Revista Saúde e Desenvolvimento Humano 7:63-72.

Lucena RGM, Barros DS, Garcia SLA, Júnior EBA, Alves LP, Diniz AFA, Alves HS, Queiroz MSR (2017) Influência da atividade física no controle da síndrome metabólica. Journal of Biology \& Pharmacy and Agricultural Management 13:22-26.

Marques JGPC, Miranda VCR, Chaves LE, Teodoro ECM (2018) Exercício aeróbico como ferramenta não farmacológica na prevenção e/ou tratamento de pacientes com síndrome metabólica. Revista Ciências e Saúde 3:22-31.

Mella A (2012) Efeitos do Exercício sobre os Fatores Determinantes da Síndrome Metabólica. Saúde em Revista 12:65-74.

Nascimento FA, Guerra RLF, Souza CAB, Silva SGA (2016) Efeitos do treinamento resistido nos parâmetros da síndrome metabólica: Uma revisão sistematizada. Revista Brasileira de Fisiologia do Exercício 15:90-101.

Oliveira CL, Fisberg M (2003) Obesidade na infância e adolescência - uma verdadeira epidemia. Arquivos Brasileiros de Endocrinologia e Metabolismo 47:107-108.

Oliveira CL, Mello MT, Cintra IP, Fisberg M (2004) Obesidade e síndrome metabólica na infância e adolescência. Revista de Nutrição 17:237-245.

Oliveira RMS, Franceschini SCC, Rosado GP, Priore SE (2009) Influência do estado nutricional pregresso sobre o desenvolvimento da síndrome metabólica em adultos. Arquivos Brasileiros de Cardiologia 92:103-108.

Paz COC, Nascimento TM, Monteiro BBS, Sousa EC, Silva DDO, Oliveira EM (2019) Influência do treinamento resistido nos critérios diagnósticos da síndrome metabólica e na capacidade funcional de mulheres idosas. Revista Brasileira de Prescrição e Fisiologia do Exercício 13:1270-1277. 
Pereira Junior M, Andrade RD, Silveira FV, Baldissera UM, Korbes AS, Navarro F (2013) Exercício físico resistido e síndrome metabólica: uma revisão sistemática. Revista Brasileira de Prescrição e Fisiologia do Exercício 7:529-539.

Rodrigues NA, Perez AJ, Pires JGP, Carletti L, Araújo MTM, Moyses MR, Bissoli NS, Abreu GR (2009) Fatores de risco cardiovasculares, suas associações e presença de síndrome metabólica em adolescentes. Jornal de Pediatria 85:55-60.

Silva BO, França MS, Oliveira RK, Silva NF, Moreira GA, Albuquerque MB, Oliveira RF, Oliveira CS, Soares V (2019) Efeitos do exercício sistematizado sobre os fatores de risco para síndrome metabólica e aptidão cardiorrespiratória: revisão sistemática. Anais da XVI Mostra Acadêmica do Curso de Fisioterapia - Centro Universitário de Anápolis 7:102-111.

Silva GAP, Balaban G, Motta MEFA (2005) Prevalência de sobrepeso e obesidade em crianças e adolescentes de diferentes condições socioeconômicas. Revista Brasileira de Saúde Materno Infantil 5:53-59.

Souza MSF, Leme RB, Franco RR, Romaldini CC, Tumas R, Cardoso AL, Damiani D (2007) Síndrome metabólica em adolescentes com sobrepeso e obesidade. Revista Paulista de Pediatria 25:214-220.

Siqueira MS, Figueiredo MPF, Lemes IR, Linares SN, Miranda RAT, Castrillón CIM, Souto LR, Souza AP, Pastre CM, Júnior JN (2016) Efeitos do treinamento resistido e aeróbio intervalado periodizados sobre o índice de massa corporal em pacientes com síndrome metabólica. Colloquium Vitae 8:22-28.

Tavares LF, Yokoo EM, Rosa MLG, Fonseca SC (2010) Síndrome metabólica em crianças e adolescentes brasileiros: revisão sistemática. Cadernos Saúde Coletiva 18:469-476.

Teixeira CGO, Silva FM, Venâncio PEM (2009) Relação entre obesidade e síndrome metabólica em adolescentes de 10 a 14 anos com obesidade abdominal. Acta Scientiarum Health Sciences 31:143-151.

Tiban RA, Prestes J (2013) Treinamento de Força e Síndrome Metabólica: uma revisão sistemática. Revista Brasileira de Cardiologia 26:66-76. 